\title{
胺磑酮、美托洛尔联合治疗急性心肌梗死合并心律失常 患者的效果
}

\author{
徐芳* \\ 宝鸡市金台医院，陕西 721000
}

摘 要: 目的: 观察胺碘酮联合酒石酸美托洛尔治疗急性心肌梗死合并心律失常患者的临床疗效。方法: 选择宝 鸡市金台医院心内科自2017年11月至2019年10月收治的200例急性心肌梗死合并心律失常患者, 分为对照组 (单纯胺 碘酮治疗) 与实验组 (胺碘酮与酒石酸美托洛尔联合治疗), 对心脏功能、心肌损伤指标、药物安全性及住院天数进 行比较。结果: 治疗后, 实验组患者心脏功能优于对照组 $(P<0.05)$, 实验组患者心肌损伤指标下降幅度大于对照 组 $(P<0.05)$, 实验组住院天数较对照组缩短 $(P<0.05)$, 两组患者并发症比较无明显差异 $(P>0.05)$ 。结论: 急性心肌梗死合并心律失常患者通过胺碘酮联合酒石酸美托洛尔联合治疗后, 患者的心脏功能提高, 心肌损伤指标下 降, 住院天数缩短, 药物安全性较高, 值得推广。

关键词：胺碘酮；酒石酸美托洛尔；急性心肌梗死合并心律失常；心脏功能

\section{一、前言}

急性心肌梗死合并心律失常作为一种常见的心内科疾病, 在中老年患者当中发病率较高, 如果不能得到及时的治 疗, 部分患者会丧失活动能力, 生活质量下降, 进而威胁生命健康。急性心肌梗死的发病原因较多且十分复杂, 过 度劳累、暴饮暴食以及情绪过激都可能引发进行心肌梗死, 进而导致血清心肌酶活性异常、心电图产生变化, 严重的 患者会因此死亡 ${ }^{[1]}$ 。近年来, 急性心肌梗死合并心律失常的发病率逐渐增多, 对于治疗方案的研究热度也逐年上升, 但是急性心肌梗死常常突然发病, 前期征兆性不强, 很容易错过最佳治疗时机。心律失常是急性心肌梗死的常见并发 症, 临床常用胺碘酮进行治疗, 然而针对胺碘酮联合酒石酸美托洛尔对急性心肌梗死合并心律失常患者治疗的有效 性、安全性进行的对比研究却比较少 ${ }^{[2]}$ 。因此, 本研究对单纯胺碘酮治疗与胺碘酮联合酒石酸美托洛尔治疗急性心肌 梗死和并心律失常患者的治疗效果进行对比分析与研究, 现报道如下。

\section{二、资料与方法}

(一) 研究对象

在征得患者及家属的同意以及医院伦理委员会批准的情况下, 选择2017年11月至2019年10月我院收治的200例急 性心肌梗死合并心律失常的患者作为研究对象, 并且排除其他疾病后, 随机将其分为实验组 100 例、对照组 100 例。

实验组中男性56例, 女性34例, 年龄为42 83岁, 平均年龄为 $(58.52 \pm 1.13)$ 岁, 心肌梗死部位: 左心室前壁26 例, 右心室前壁20例, 左心室后壁26例, 右心室后壁28例, 心脏功能分级：一级54例, 二级34例, 三级12例。

对照组中男性55例, 女性35例, 年龄在43 82岁, 平均年龄为 $(58.69 \pm 1.15)$ 岁, 心肌梗死部位: 左心室前壁28 例，右心室前壁26例，左心室后壁20例，右心室后壁26例，心脏功能分级：一级52例，二级34例，三级14例。

据临床资料显示, 两组患者年龄、性别、身高、体重、病程、文化程度等一般资料差异无统计学意义 $(P>$ $0.05)$ 。

\section{（二）纳人标准及排除标准}

1. 纳人标准

( 1 ) 符合 《内科疾病诊断标准》 ${ }^{[3]}$ 《急性ST段抬高型心肌梗死诊断和治疗指南》 (2015) ${ }^{[4]}$ 相关标准, 诊断为急 性心肌梗死合并心律失常。

（2）患者耐受胺碘䣳联合酒石酸美托洛尔药物联合治疗，无不良反应。

*通讯作者: 徐芳, 1985年6月, 女, 汉族, 陕西宝鸡人, 就职于宝鸡市金台医院心内科临床工作, 主治医师, 硕 士研究生。研究方向: 慢性心力衰竭。 
（3）自我意识清晰，能够与医务人员正常交流。

2. 排除标准

（1）认知功能异常、凝血异常、患有恶性肿瘤。

(2) 精神异常或者其他脏器功能受损患者。

(3) 妊娠期、哺乳期患者。

(4) 慢性心肌梗死患者。

(三) 方法

两组患者住院后都给予常规基础治疗，合并有高血压、糖尿病的患者，给予控制血压、血糖等治疗。

1. 对照组

对照组患者采取单纯胺碘酮片（上海上药信宜药厂, 规格： $0.2 \mathrm{~g}$, 批准文号：国药准字H31021872) 治疗, 每次 服用胺碘酮片 $200 \mathrm{mg}$, 每天三次, 连续服用七天; 第八天开始改为每次服用胺碘酮片 $200 \mathrm{mg}$, 每天二次, 连续服用七 天；第十五天开始改为每次服用胺碘酮片 $200 \mathrm{mg}$ ，每天一次，连续服用七天；连续治疗一个疗程共计二十一天。

2. 实验组

实验组患者采取胺碘酮联合酒石酸美托洛尔 ( 常州四药制药有限公司, 规格： $25 \mathrm{mg}$, 批准文号：国药准字 $\mathrm{H} 32025169$ ) 治疗, 在对照组基础上每次增加服用酒石酸美托洛尔 $6.25 \mathrm{mg}$, 每天两次, 连服七天; 第八天开始每次服 用酒石酸美托洛尔 $12.5 \mathrm{mg}$, 每天两次, 并结合患者恢复程度以及心脏功能水平调整用药剂量, 最大幅度不超过每天 $200 \mathrm{mg}$, 连续治疗一个疗程及二十一天。

( 四) 评价指标

1. 心脏功能

两组患者治疗前后均采用超声心动图测量患者左心室射血分数（LVEF）、心输出量（CO ）以及左心室舒张末期 内径 (LVEDD)，并记录住院天数。

2. 心肌损伤指标

对比治疗前后三周两组患者的肌酸激酶同工酶、肌酸激酶以及脑钠肽、N末端脑钠肽前体水平。

3. 药物安全性

记录并比较两组患者治疗过程中不良症状的发生率。

(五) 统计学方法

研究获取患者资料采用SPSS18.0系统软件分析; 计量资料用 $(\bar{x} \pm s)$ 表示，并用 $t$ 检验; 计数资料用 $[n(\%)]$ 表 示，并用 $x^{2}$ 检验； $P<0.05$ 表示有统计学意义。

\section{三、结果}

（一）两组患者心脏功能及住院时间的对比

治疗之前, 实验组与对照组相比心脏功能, 差异不具有统计学意义 $(P>0.05)$, 治疗之后, 实验组患者左心室 射血分数 $(\mathrm{LVEF})$ 水平下降幅度大于对照组 $(P<0.05)$, 实验组患者心输出量 $(\mathrm{CO})$ 水平以及左心室舒张末期内径 ( LVEDD ) 水平上升幅度高于对照组 $(P<0.05)$ ，差异具有统计学意义，具体数据如表1所示。

表1 两组患者心脏功能及住院时间比较 $(\overline{\boldsymbol{x}} \pm \boldsymbol{s})$

\begin{tabular}{|c|c|c|c|c|c|c|c|c|}
\hline \multirow[t]{2}{*}{ 组别 } & \multirow[t]{2}{*}{ 例数 } & \multicolumn{2}{|c|}{ 左心室射血分数LVEF（％） } & \multicolumn{2}{|c|}{ 心输出量CO（L/min） } & \multicolumn{2}{|c|}{$\begin{array}{c}\text { 左心室舒张末期内径LVEDD } \\
(\mathrm{mm})\end{array}$} & \multirow{2}{*}{$\begin{array}{l}\text { 住院时间 } \\
\text { (d) }\end{array}$} \\
\hline & & 治疗前 & 治疗后 & 治疗前 & 治疗后 & 治疗前 & 治疗后 & \\
\hline 对照组 & 100 & $37.5 \pm 2.1$ & $44.2 \pm 3.1$ & $2.6 \pm 0.3$ & $3.6 \pm 0.4$ & $66.2 \pm 4.1$ & $55.3 \pm 2.7$ & $45.6 \pm 2.4$ \\
\hline 实验组 & 100 & $37.6 \pm 2.2$ & $53.9 \pm 2.9$ & $2.5 \pm 0.6$ & $4.6 \pm 0.2$ & $66.1 \pm 4.2$ & $39.5 \pm 2.8$ & $36.4 \pm 2.8$ \\
\hline$t$ & - & 1.245 & 2.315 & 2.656 & 1.248 & 1.224 & 2.357 & 2.645 \\
\hline$P$ & - & $>0.05$ & $<0.05$ & $>0.05$ & $<0.05$ & $>0.05$ & $<0.05$ & $<0.05$ \\
\hline
\end{tabular}

(二) 两组患者心肌损伤指标对比

治疗之前, 实验组与对照组相比心肌损伤指标, 差异不具有统计学意义 $(P>0.05)$, 治疗之后, 实验组患者肌 
酸激酶同工酶、肌酸激酶以及脑钠肽、 $\mathrm{N}$ 末端脑钠肽前体水平下降幅度大于对照组 $(P<0.05)$, 差异具有统计学意 义，具体数据如表2所示。

表2 两组患者心肌损伤指标比较 $(\%)$

\begin{tabular}{|c|c|c|c|c|c|c|c|c|c|}
\hline \multirow[t]{2}{*}{ 组别 } & \multirow[t]{2}{*}{ 例数 } & \multicolumn{2}{|c|}{ 肌酸激酶同工酶（U/L ） } & \multicolumn{2}{|c|}{ 肌酸激酶 ( U/L ) } & \multicolumn{2}{|c|}{ 脑钠肽（pg/mL） } & \multicolumn{2}{|c|}{$\begin{array}{c}\mathrm{N} \text { 末端脑钠肽前体水平 } \\
(\mathrm{pg} / \mathrm{mL})\end{array}$} \\
\hline & & 治疗前 & 治疗后 & 治疗前 & 治疗后 & 治疗前 & 治疗后 & 治疗前 & 治疗后 \\
\hline 实验组 & 100 & $2.2 \pm 1$ & $2.0 \pm 2$ & $960 \pm 3$ & $881 \pm 2$ & $2.5 \pm 1$ & $2.2 \pm 3$ & $759 \pm 2$ & $571 \pm 2$ \\
\hline 对照组 & 100 & $2.3 \pm 1$ & $1.2 \pm 2$ & $959 \pm 2$ & $720 \pm 1$ & $2.6 \pm 2$ & $1.5 \pm 2$ & $760 \pm 1$ & $452 \pm 3$ \\
\hline$t$ & - & 1.25 & 2.35 & 1.64 & 2.31 & 1.68 & 2.67 & 1.54 & 2.67 \\
\hline$P$ & - & $>0.05$ & $<0.05$ & $>0.05$ & $<0.05$ & $>0.05$ & $<0.05$ & $>0.05$ & $<0.05$ \\
\hline
\end{tabular}

(三) 两组患者用药安全性比较

用药后，两组患者并发症比较，差异不具有统计学意义 $(P>0.05)$ ，如下表3所示。

表3 两组患者用药后并发症比较

\begin{tabular}{|c|c|c|c|c|c|c|c|c|c|c|c|}
\hline \multirow{2}{*}{ 组别 } & \multirow{2}{*}{ 例数 } & \multicolumn{2}{|c|}{ 胃肠道反应 } & \multicolumn{2}{|c|}{ 血压波动 } & \multicolumn{2}{|c|}{ 肝肾功能异常 } & \multicolumn{2}{|c|}{ 头晕头痛 } & \multicolumn{2}{|c|}{ 其他 } \\
\hline & & 例数 & $\%$ & 例数 & $\%$ & 例数 & $\%$ & 例数 & $\%$ & 例数 & $\%$ \\
\hline 对照组 & 100 & 6 & 6 & 0 & 0 & 4 & 4 & 7 & 7 & 3 & 3 \\
\hline 实验组 & 100 & 4 & 4 & 1 & 1 & 3 & 3 & 8 & 8 & 1 & 1 \\
\hline$\chi^{2}$ & - & \multicolumn{2}{|c|}{1.978} & \multicolumn{2}{|c|}{2.015} & \multicolumn{2}{|c|}{1.568} & \multicolumn{2}{|c|}{2.647} & \multicolumn{2}{|c|}{1.678} \\
\hline$P$ & - & \multicolumn{2}{|c|}{$>0.05$} & \multicolumn{2}{|c|}{$>0.05$} & \multicolumn{2}{|c|}{$>0.05$} & \multicolumn{2}{|c|}{$>0.05$} & \multicolumn{2}{|c|}{$>0.05$} \\
\hline
\end{tabular}

四、讨论

急性心肌梗死是心内科常见的疾病, 近几年发病率逐年上升, 心律失常是其常见的并发症, 引起了医学界的广 泛关注 ${ }^{[3]}$ 。传统急性心肌梗死合并心律失常采用单纯胺碘酮治疗, 作为一种常用的急性心肌梗死合并心律失常治疗手 段, 治疗效果不佳, 近几年通过医学研究发现胺碘酮联合酒石酸美托洛尔能够有效缩短患者的住院时间, 并显著提升 治疗效果 ${ }^{[4-5]}$ 。

作为临床上发生率较高的心血管系统疾病, 急性心肌梗死与心律失常同时叠加发生的概率非常高, 在发生后, 两 种疾病相互补充, 患者的死亡率明显提升。为了缓解急性心肌梗死并发心律失常患者的病情, 临床通常采用溶栓治 疗, 但是治标不治本, 只能改善患者预后, 并且, 部分患者在接受溶栓治疗后受到严重心律失常的影响, 溶栓效果不 显著 ${ }^{[6-7]}$ 。作为人体重要的器官, 心脏肌肉发达, 收缩能力较强, 通过血管壁的收缩完成血液的输送 ${ }^{[8]}$ 。心脏只有正常 的收缩, 才能保证血液循环的正常进行, 但是心肌梗死合并心律失常患者的血管堵塞, 并且心脏节律的失常, 会导致 心脏功能的下降, 长时间还会造成严重的心肌损伤。由于胺碘酮的半衰期较长, 在心肌梗死合并心律失常患者的治疗 中能够抑制心房、心肌传导纤维的快钠离子内流, 从而使窦房结的自律性得到改善 ${ }^{[9]}$ 。另外, 经过近代医学研究结合 药理结果表明，胺碘酮联合酒石酸美托洛尔治疗的效果更佳 ${ }^{[10]}$ 。

本次研究结果发现, 治疗之前, 实验组与对照组相比心脏功能, 差异不具有统计学意义 $(P>0.05)$, 治疗之 后, 实验组患者左心室射血分数 ( LVEF) 水平下降幅度大于对照组, 实验组患者心输出量 $(\mathrm{CO})$ 水平以及左心室舒 张末期内径 $($ LVEDD ) 水平上升幅度高于对照组 $(P<0.05)$, 治疗之后, 实验组患者肌酸激酶同工酶、肌酸激酶以 及脑钠肽、 $\mathrm{N}$ 末端脑钠肽前体水平下降幅度大于对照组 $(P<0.05)$, 用药后, 两组患者并发症比较, 差异不具有统计 学意义 $(P>0.05)$ 。

综上所述, 相较于单纯胺碘酮治疗, 在接受胺碘酮联合酒石酸美托洛尔治疗之后, 急性心肌梗死合并心律失常患 者心脏功能明显得到提升，心肌损伤得到明显改善，无明显并发症，并且患者的住院时间显著缩短，值得临床推广。

\section{参考文献:}

[1]贝正平.内科疾病诊断标准[M].北京:科学出版社, 2001:6.

[2]中华医学会心血管病学分会, 中华心血管病杂志编辑委员会.急性ST段抬高型心肌梗死诊断和治疗指南[J]. 中华心 血管病杂志, 2015,43(5):380-393.

[3]李国超.注射用环磷腺苷葡胺与琥珀酸美托洛尔联合胺碘酮治疗急性心肌梗死合并心律失常的临床效果 [J]. 河南 
医学研究, 2020,29(19):3569-3570.

[4]褚龙.分析急性心肌梗死合并室性心律失常采用酒石酸美托洛尔联合盐酸胺碘酮治疗的效果 [J].中外医疗, 2020,39(10):100-101+104.

[5]王尚华, 关亚东.门冬氨酸钾镁联合酒石酸美托洛尔注射液与盐酸胺碘酮治疗急性心肌梗死合并心律失常临床观 察[J].社区医学杂志, 2019,17(23):1522-1524+1528.

[6]李西红,孙黎博.胺碘酮联合美托洛尔治疗急性心肌梗死合并心律失常患者的效果及对患者心脏功能的影响分析 [J].山西医药杂志, 2019,48(22):2734-2737.

[7]张建新, 胡大军. 环磷腺苷葡胺与琥珀酸美托洛尔联合胺碘酮治疗急性心肌梗死心律失常的疗效 [J]. 当代医学, 2019,25(29):23-25.

[8]黄涌.胺碘酮联合美托洛尔治疗心肌梗死后室性心律失常的效果[J].中国民康医学, 2019,31(19):3-5.

[9]吴艳丽, 路素品.美托洛尔联合胺碘酮治疗急性心肌梗死并发快速性心律失常 [J]. 实用中西医结合临床, 2019,19(08):67-68.

[10]连会军.胺碘酮联合美托洛尔治疗急性心肌梗死合并心律失常患者的效果及对患者心脏功能的影响 [J].中国民间 疗法, 2019,27(12):75-77. 